\title{
Impact des activités anthropiques sur la diversité ligneuse et la structure de Vitellaria paradoxa Gaertn. C.F. dans le Chantier d'Aménagement Forestier de Cassou (Burkina Faso)
}

\author{
Received: 2019-09-10; revised: 2019-11-20; accepted: 2019-11-22
}

François Wenemi Kagambèga ${ }^{1, *}$, Nouhoun Zampaligre², Didier Zida ${ }^{1}$, et Louis Sawadogo ${ }^{1}$

\footnotetext{
1. Centre National de la Recherche Scientifique et Technologique, Institut de l'environnement et de Recherches Agricoles, Département Environnement et Forêts, 03 BP 7047, Ouagadougou 03, Burkina Faso

2. Centre National de la Recherche Scientifique et Technologique, Institut de l'environnement et de Recherches Agricoles, Station de Farako-ba, 01 BP 910 Bobo Dioulasso 01, Burkina Faso

* Corresponding author. E-mail address: wenemifrancois@yahoo.fr
}

\begin{abstract}
Résumé: Au Burkina Faso, les unités d'aménagements forestiers (UAFs) sont confrontées à une forte pression anthropique qui cause la dégradation des ressources ligneuses dont Vitellaria paradoxa est l'une des espèces surexploitées. Afin de contribuer à la gestion durable de ces UAFs, il s'avère nécessaire d'analyser l'impact des pressions anthropiques sur la phytodiversité et la dynamique de Vitellaria paradoxa. Ainsi, des facteurs tels que l'agriculture, la coupe illégale de bois, le pâturage et les feux non autorisé ont été évalués dans l'UAF 2, l'UAF B et l'UAF C, où des inventaires forestiers ont été conduits. Les résultats montrent que le pâturage et les feux non autorisé ont été les facteurs les plus fréquents dans les UAFs, surtout dans l'UAF C (>90\%) alors que les autres facteurs ont été plus abondants dans l'UAF 2 . La phytodiversité mesurée est presque similaire dans les trois UAFs avec toutefois des indices de diversité plus élevés dans l'UAF 2. La structure de Vitellaria paradoxa montre une prédominance d'individus jeunes dans les UAFs et des densités plus élevées dans l'UAF C tandis que sa régénération est plus importante dans l'UAF 2. L'état sanitaire de l'espèce est globalement satisfaisant bien que la principale menace soit l'infestation des Loranthaceae qui a été plus importante dans l'UAF 2 (7\%). L'exploitation durable des UAFs nécessite la mise en œuvre d'un programme de conservation avec une forte implication des populations riveraines.
\end{abstract}

Mots clés: Aménagement forestier, Burkina Faso, diversité floristique, dynamique structurale, Vitellaria paradoxa.

\section{EFFECT OF ANTHROPOGENIC ACTIVITIES ON WOODY SPECIES DIVERSITY AND STRUCTURE OF VITELLARIA PARADOXA GAERTN. C. F. IN THE FOREST MANAGEMENT DOMAIN OF CASSOU, BURKINA FASO}

Summary: In Burkina Faso, forest management units (FMUs) are facing to the intensification of anthropogenic pressures which causes the degradation of the woody resources of which Vitellaria paradoxa is one of the overexploited. To support their sustainable management, the impacts of human pressure on the diversity and the structural dynamic of $V i$ tellaria paradoxa need to be analysed. Therefore, anthropogenic factors such as practice of agriculture, logging, grazing and fire were assessed at Cassou in FMU 2, FMU B and FMU C, in which forest inventories were conducted. The results show that grazing and fires were the most frequent factors in FMUs, especially in FMU C (>90\%) while the other factors were more observed in FMU 2. Phytodiversity is almost similar in the whole FMUs, even if FMU 2 presents the higher diversity indices. Structural analysis of Vitellaria paradoxa showed a predominance of young individuals in the FMUs and a higher tree density in the FMU C whereas tree regeneration was greater in the FMU 2. The health status of the species is overall satisfactory although the main threat is the Loranthaceae infestation which is more important in FMU 2 (7\%). The sustainable exploitation of FMUs requires an implementation of a conservation program with a strong involvement of local populations.

Key words: Forest management, Burkina Faso, floristic diversity, structural dynamics, Vitellaria paradoxa. 


\section{INTRODUCTION}

Les écosystèmes forestiers et savanicoles jouent un rôle fondamental dans la satisfaction des besoins de base des populations du continent africain. En Afrique subsaharienne, près de 600 millions de personnes dépendent de ces écosystèmes pour leur nourriture et / ou leurs revenus (KoFFI \& al. 2016). Cependant, les multiples usages concurrentiels des ressources de ces formations végétales, sont également sources d'externalités négatives conduisant à leur dégradation ou à la déforestation. Effet, depuis plusieurs décennies, les écosystèmes forestiers subissent une forte dégradation, entrainant leurs dysfonctionnements et des pertes de biodiversité (FAO 2003). Au Burkina Faso, les facteurs majeurs de dégradation des ressources forestières sont les feux de brousse, la coupe abusive du bois, le surpâturage, la mortalité naturelle des espèces due aux aléas climatiques et les défrichements agricoles anarchiques (MEDD 2011 ; NACOULMA \& al. 2011). Les recherches effectuées et les données disponibles révèlent une amplification de la déforestation (FAO 2015) qui se manifeste entre autres à travers la dégradation de la couverture végétale, la dégradation des sols, la dégradation des ressources en eau et des habitats de la faune (ETONGO \& al. 2018).

Dans les parties nord et centrale du pays, les formations naturelles sont dans un état de dégradation avancé entraînant une forte migration du Nord vers le Sud et l'Est du pays (SAWAdogo 2007). Une pression de plus en plus exacerbée s'exerce alors sur les ressources naturelles, autres fois abondantes, de ces zones d'accueil notamment au Sud du pays. En effet, la croissance démographique contraint les paysans à rechercher de nouvelles terres agricoles au détriment des milieux naturels et entraîne également une consommation et une perturbation plus importante des ressources végétales (Belem \& al. 2018). Les Chantier d'Aménagement Forestiers (CAF) installées au Sud du pays depuis les années 1980, pour assurer une gestion durable des ressources naturelles (Coulibaly-Lingani \& al. 2011), n'échappent pas, eux aussi à toutes ces menaces. Bien que le modèle des CAF fonctionne sur la base d'un plan d'aménagement et de gestion approuvée, des pressions exceptionnelles et très intenses sont exercées sur leurs ressources forestières (BousSIM \& al. 2009). Des études antérieures dans ces CAF, ont porté sur les défis de la gestion décentralisée des forêts, les facteurs influant sur l'accès aux produits forestiers, la gestion participative ou conjointe des forêts aménagées (BoudA et al. 2011; Coulibaly-Lingani \& al. 2011). Aussi, des études récentes, suscite des inquiétudes quant à la durabilité de ces CAF au regard des besoins grandissants des populations humaines (AREVALO 2016) et de la forte pression anthropique exercée sur un certain nombre d'espèces surexploitées (TANYI \& al. 2018). En effet, ces fortes pressions peuvent conduire à une modification significative de la structure de la végétation avec comme conséquence la disparition ou la régression de certaines espèces ligneuses utiles pour les populations (Lessmeister \& al. 2015 ; SANDJONG \& al. 2018). Cependant, des données concernant les caractéristiques structurales et la dynamique des espèces végétales en général et des espèces surexploitées en particulier, ne sont pas suffisamment documentées. L'une des espèces ligneuses les plus importantes au Burkina Faso d'un point de vue socioéconomique est Vitellaria paradoxa (RABIOU Y al. 2016) dont les utilisations sont multiples et diverses (LESSMEISTER \& al. 2015).

La compréhension de la dynamique de cette espèce très exploitée dans les chantiers d'aménagement forestiers (CAF) est primordiale pour sa conservation durable. Il est donc indispensable d'analyser l'état de la diversité floristique des ligneux et la structure de cette espèce dans les Unités d'Aménagement Forestiers (UAFs) du CAF pour mieux appréhender le niveau de menace et proposer des stratégies de gestion durable. L'objectif général de la présente étude est de décrire la diversité de la flore ligneuse et d'analyser les attributs structuraux des populations de Vitellaria paradoxa dans trois UAFs du CAF de Cassou, soumis à différents degrés de perturbations anthropiques. Les objectifs spécifiques sont : (i) d'évaluer l'incidence et l'ampleur des facteurs anthropiques courants (activités agricoles, coupes illégales de bois, feux non autorisés et pâturage) dans les UAFs ciblées ; (ii) d'analyser l'impact de ces facteurs anthropique sur les caractéristiques floristiques de la végétation ligneuse dans ces UAFs; (iii) d'établir la distribution structurale des populations de Vitellaria paradoxa et de caractériser leur tendance évolutive. L'étude se fonde sur les hypothèses suivantes : (H1) La végétation des UAFs proches de l'unité de gestion basée à Cassou, est moins perturbée que celle des UAFs éloignées ; (H2) la structure démographique des peuplements de Vitellaria paradoxa au sein des UAFs est fonction d'un gradient d'anthropisation avec une dynamique structurale stable dans les UAFs proches de l'unité de gestion. Les résultats obtenus permettront de disposer d'indicateurs nécessaires à l'élaboration de stratégies de gestion durable de ces ilots forestiers en vue d'optimiser la contribution de ces ressources forestières à l'amélioration des conditions socioéconomiques des populations.

\section{Materiel et Methodes}

\subsection{Présentation de la zone d'étude}

L'étude a été menée dans le Chantier d'Aménagement Forestier $(\mathrm{CAF})$ de Cassou, situé dans la province du Ziro, à $35 \mathrm{~km}$ de Sapouy son chef-lieu (Fig. 1). Localisé dans le domaine phytogéographique Sud-soudanien entre les latitudes $12^{\circ} 24^{\prime}$ et $12^{\circ}$ Nord et les longitudes $2^{\circ} 39^{\prime}$ et $2^{\circ}$ Ouest), le CAF de Cassou est sous l'influence d'un climat soudanien caractérisé par une longue saison sèche et une saison pluvieuse qui dure généralement moins de 5 mois (de mai à septembre / octobre). Les précipitations annuelles peuvent atteindre ou même dépasser $800 \mathrm{~mm}$ suivant les années. Avec une température moyenne de $31,5^{\circ} \mathrm{C}$, le mois d'Avril est le plus chaud de l'année pendant que Décembre est le mois le plus froid avec une température moyenne de 25,2 ${ }^{\circ} \mathrm{C}$. La zone appartient au vaste complexe du plateau mossi mais présente un relief peu accidenté se caractérisant par une vaste pénéplaine. Les sols sont de types argileuxsableux, limoneux ou gravillonnaire et sont représentés par les Cambisols, les Lixisols et les Luvisols. 


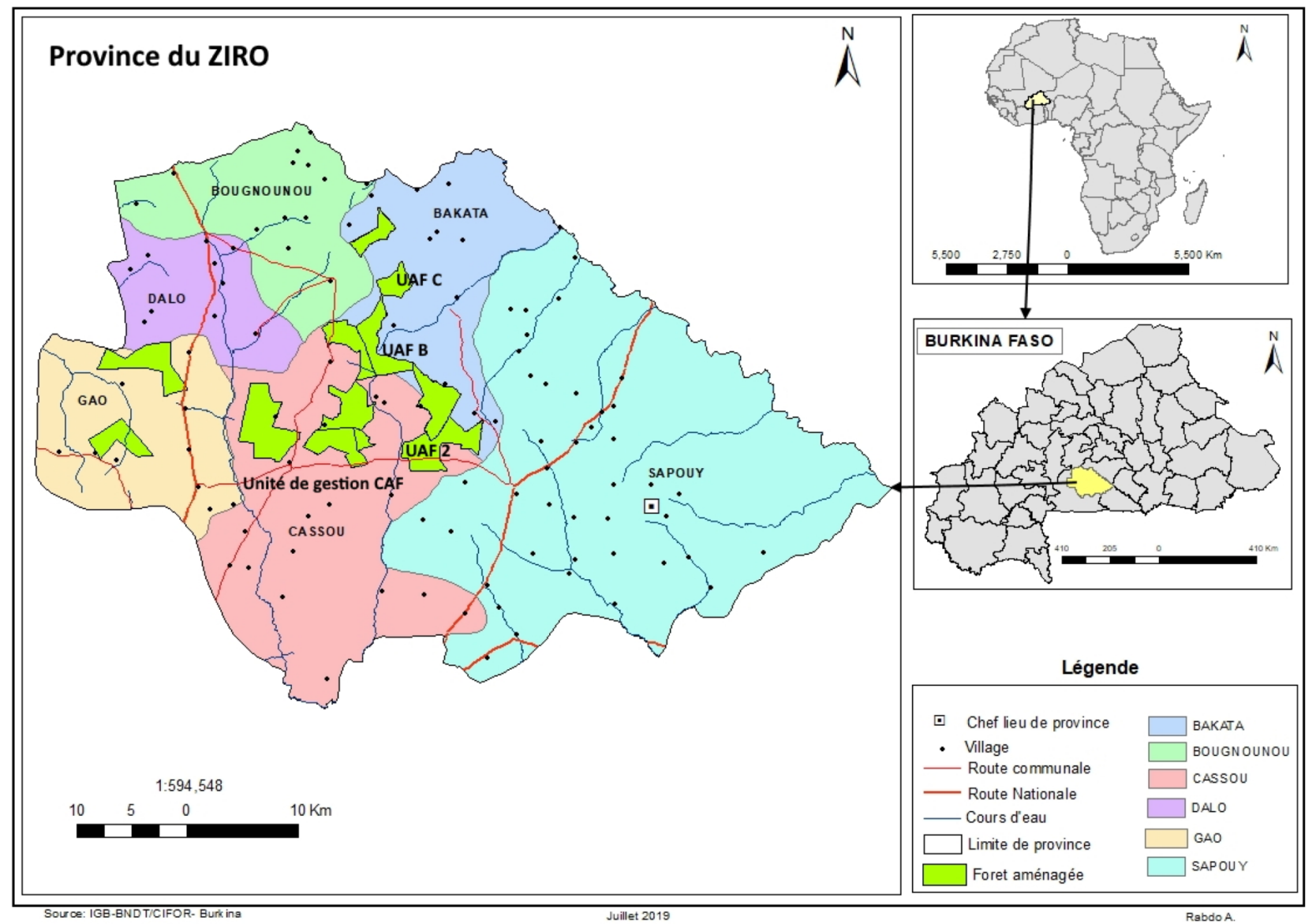

Fig. 1: Localisation du CAF de Cassou et des UAFs étudiées / Location of Cassou's FMD and the studied FMUs.

\subsection{Techniques d'aménagement utilisées dans le CAF de Cassou}

D'une superficie de 29515 ha, le CAF de Cassou fait partie d'un ensemble de sept (7) CAF du Burkina Faso créés en 1986 dans un programme d'aménagement des formations naturelles sur financement PNUD avec une implication de la FAO (ETONGO \& al. 2018). L'objectif principal des CAF était de réguler l'exploitation du bois-énergie au profit des villages environnements et des centres urbains du pays. Le CAF de Cassou qui a été créé en 1991, est divisée en unités et sous-unités par les acteurs en charges de sa gestion. Ainsi, il compte neuf Unités d'Aménagement Forestiers (UAFs) et trois sous-unités. Chacune des unités / sousunités est subdivisée en quinze parcelles exploitées successivement chaque année avec un cycle de rotation de 15 ans. L'exploitation forestière des parcelles est réalisée selon un plan d'aménagement avec un cahier de charge et suit le schéma des prescriptions suivantes:

- une coupe sélective de $50 \%$ du volume de bois commercialisable sur pied;

- l'utilisation du feu précoce annuel sauf sur les parcelles nouvellement coupées ;

- la protection des parcelles exploitées du feu et du pâturage pendant 3 à 5 ans ;
- l'interdiction du pâturage dans les forêts classées non aménagées, les parcs nationaux et les ranchs de gibier ;

- l'enrichissement par semis direct et par plantation des espaces exploitées et des zones dénudées avec des espèces locales.

Les critères de coupe appliqués dans le CAF sont respectivement :

- la densité du peuplement, l'exploitation étant interdite dans les sites présentant moins de 200 pieds/ha ;

- l'état sanitaire, qui privilégie prioritairement la coupe des arbres malades ou malformés ; les considérations écologiques, qui épargnent les espèces protégées ou rares et les semenciers ;

- les dimensions de commercialisation qui orientent l'exploitation sur les arbres dont le diamètre à hauteur de poitrine est compris entre 10 et $25 \mathrm{~cm}$;

- la hauteur de coupe fixée à $15 \mathrm{~cm}$ maximum pour permettre l'émergence de rejets de souche le plus proche du sol. 


\subsection{Collecte de données}

\subsubsection{Echantillonnage et installation des placettes}

Le CAF de Cassou a été choisie en raison de la présence d'une unité de gestion toujours en activité, ce qui constitue une meilleure opportunité pour évaluer l'efficacité des approches de gestion mises en œuvre. Pour caractériser l'état actuel de diversité du peuplement ligneux du CAF, un inventaire floristique a été réalisé dans trois (03) Unités d'Aménagement Forestiers (UAFs) sur un total de douze UAFs que compte le CAF de Cassou (Fig. 1). Ces UAFs (UAF 2, UAF B et UAF C) ont été échantillonnées selon des critères tels que l'accessibilité, la représentativité et l'homogénéité, à partir d'une localisation à l'aide de cartes d'occupation du sol préexistantes. L'étude a été conduite sur l'ensemble des 15 parcelles de chacune des UAFs ciblées où un échantillonnage aléatoire et stratifié aux taux de $0,3 \%$ a été adopté ; l'unité d'échantillonnage qui représente un relevé floristique est une placette circulaire de $20 \mathrm{~m}$ de rayon $\left(1256,64 \mathrm{~m}^{2}\right)$. L'installation des placettes a tenu compte de la taille des UAFs comme cela est présenté dans le Tableau 1. Sur cette base, les points représentant les placettes de chaque parcelle dans les différentes UAFs ont été pré-positionnés de façons aléatoires sur les cartes géo-référencier des UAFs et les coordonnées géographiques reportés dans des GPS. Les déplacements pour l'implantation des placettes d'inventaire sur le terrain se sont effectués à l'aide de ces GPS, à travers les pistes existantes.

Tableau 1: Caractéristiques des UAFs étudiées et répartition du nombre de placettes / Characteristics of the studied FMUs and distribution of the number of plots.

\begin{tabular}{|l|l|l|l|}
\hline Caractéristiques des UAFs & UAF 2 & UAF B & UAF C \\
\hline Quelques villages associés & $\begin{array}{l}\text { Luin, } \\
\text { Vrassan, } \\
\text { Diao }\end{array}$ & Kou & $\begin{array}{l}\text { Bakata, } \\
\text { Lorou, } \\
\text { Zenloua }\end{array}$ \\
\hline $\begin{array}{l}\text { Distance avec l'unité de gestion } \\
\text { (Km) }\end{array}$ & 10 & 14 & 18 \\
\hline Superficie (ha) & 3774 & 2603 & 1214 \\
\hline $\begin{array}{l}\text { Superficie moyenne de la parcelle } \\
\text { (ha) }\end{array}$ & 251,6 & 173,53 & 80,93 \\
\hline Nombre de placettes / parcelle & 8 & 6 & 3 \\
\hline Nombre total de placettes & 120 & 90 & 45 \\
\hline
\end{tabular}

\subsubsection{Caractérisation de la pression anthropique}

Les facteurs de perturbations anthropiques tels que feux non autorisés, les activités agricoles, le pâturage et la coupe frauduleuse de bois, ont été caractérisés dans chaque placette. La pression anthropique est considérée nulle dans une placette, lorsqu'il n'y a ni trace de feu, ni trace de pâture d'animaux domestiques (présence d'animaux en pâture, de déjections, de traces de piétinements, etc.), ni activité agricole, ni trace de coupe illégale de bois. Par contre, lorsque dans une placette, on observe l'un de ces phénomènes, c'est leur intensité qui détermine le degré de l'action anthropique. Ainsi la pression anthropique a été évaluée à l'échelle de chaque placette selon une codification à quatre niveaux $(0$, 1,2 et 3) pour chaque facteur :

- lorsqu'aucune trace de perturbations anthropiques relatives aux facteurs ci-dessus énumérés n'est observée dans une placette, le code 0 est attribué ;
- lorsque les traces sont faibles, le code 1 est attribué;

- le code 2 est attribué lorsque le degré de perturbation est moyen;

- lorsque les traces de perturbation sont élevées, le code 3 est attribué.

\subsubsection{Inventaire floristique et mesures dendrométriques}

Pour caractériser la diversité floristique du peuplement ligneux et la structure de Vitellaria paradoxa dans les différentes UAFs, un inventaire floristique a été conduite et des mesures dendrométriques réalisées sur les peuplements de Vitellaria paradoxa. L'unité d'échantillonnage a été une placette circulaire de $20 \mathrm{~m}$ de rayon, soit une aire de relevée de 1256,64 $\mathrm{m}^{2}$. Dans chaque placette, tous les individus ligneux adultes (de diamètre à hauteur de poitrine $(\mathrm{DBH})$ $>5 \mathrm{~cm}$ ) sont inventoriés. L'inventaire floristique a été faite de façon exhaustive dans les différentes placettes et pour chaque individu identifié, les paramètres suivant sont noté sur une fiche de relevés: le DBH, la hauteur et l'état sanitaire. Les mesures de DBH ont été faites à l'aide d'un mètre ruban souple pendant qu'un dendromètre électronique associé au télémètre laser ont servi à l'estimation du de la hauteur des arbres, depuis la base du pied jusqu'au bout de la branche la plus élevée. L'état sanitaire des individus de Vitellaria paradoxa a été évalué suivant les codifications définies par KaBORE (2004) et contenues dans le tableau 2.

Tableau 2: Code d'appréciation de l'état sanitaire des espèces ligneuses / Code of assessment of the woody species' health status.

\begin{tabular}{|l|l|}
\hline Code & Signification \\
\hline 1 & Individu sans défaut visible \\
\hline 2 & Individu ébranché \\
\hline 3 & Individu brulé (présence de trou ou de crevasse dans le bois) \\
\hline 4 & Individu semi-mort ou cime plus ou moins desséchée \\
\hline 5 & Individu mort sur pied \\
\hline 6 & Individu parasité par des Loranthacées \\
\hline
\end{tabular}

Dans chaque placette principale, deux sous-placettes de 10 $\mathrm{m}^{2}$ chacun $(5 \mathrm{~m} \times 2 \mathrm{~m})$, sont installées pour l'évaluation de la régénération, par l'identification et le comptage de tous les individus de $\mathrm{DBH} \leq 5 \mathrm{~cm}$ (Ouedraogo \& al. 2006). L'identification des échantillons d'espèces récoltées sur le terrain a été faite par comparaison avec les spécimens de l'Herbier National du Burkina Faso. La classification APG III (2009) a été utilisée pour la nomenclature botanique des familles.

\subsection{Analyse et traitement des données}

\subsubsection{Pression anthropique}

La pression anthropique est évaluée pour chaque facteur en considérant la proportion des placettes concernées par le facteur de perturbation codifié par rapport au nombre total de placettes inventoriées dans l'UAF selon la formule 1:

Formule 1:

Perturbation anthropique $(\%)=\frac{\text { Nombre de placettes codifiées i }}{\text { Nombre total de placettes inventoriée }} \times 100$

Où i représente le niveau du code $(0,1,2$ ou 3$)$ pour le facteur concerné. 


\section{4. 2 Données floristiques et dendrométriques}

À partir des données collectées sur le terrain, la richesse et la diversité floristique des peuplements ont été calculées dans les trois (3) Unités d'Aménagement Forestier (UAFs). Ainsi, tous les individus rencontrés lors de l'inventaire ont été regroupés par espèce, genre et famille. Pour mettre en évidence les espèces et les familles dominantes dans chaque UAF, l'Indice de Valeur d'Importance (IVI) de CURTIS \& MCintosh (1950) des espèces et la Valeur d'Importance de chaque Familles (FIV) ont été déterminés à partir des paramètres suivants :

- La densité (nombre d'individus d'une espèce par unité de surface $(\mathrm{ha})$ ) et sa valeur relative $=$ (nombre d'individus de cette espèce / nombre total des individus) x 100 ;

- La dominance d'une espèce (somme des surface terrière par unité de surface (ha) $)$ et sa valeur relative $=($ Surface terrière totale d'une espèce / Surface terrière totale de toutes les espèces) x 100 ;

- La fréquence de chaque espèce (dans les placettes inventoriées dans chaque UAF) et sa valeur relative $=($ Fréquence d'une espèce $/$ Somme de toutes les fréquences) x 100 ;

- La densité relative de chaque famille ((nombre d'individus de cette famille / nombre total des individus) $\mathrm{x} 100$ ) et leur dominance relative $=$ Surface terrière totale d'une famille / Surface terrière totale de toutes les familles) x 100 ;

- $\quad$ La diversité relative de chaque famille $=$ (nombre d'espèce dans une famille / nombre total d'espèces) $\mathrm{x} 100$.

L'Indice de Valeur d'Importance (IVI) correspond à la somme de la densité relative, de la dominance relative et de la fréquence relative. La valeur d'importance d'une famille (FIV) est la somme de la densité relative, de la dominance relative et de la diversité relative de chaque famille (MoRI et al. 1983)

L'évaluation de la diversité biologique s'est faite par le calcul des indices suivant :

- la richesse spécifique (S) qui est le nombre total d'espèces que comporte le peuplement considéré dans un écosystème donné ;

- l'indice de Shannon-Weaver qui permet de quantifier l'hétérogénéité de la diversité spécifique par la formule $: H^{\prime}=-\sum P_{i} \log _{2} P_{i}$ où $P_{i}$ est la Proportion d'une espèce i par rapport au nombre total d'espèces (S) dans le milieu (DAJOZ 2008);

- l'indice de Simpson (D) qui donne la probabilité pour que deux individus sélectionnés au hasard dans une population appartiennent à la même espèce: $\mathrm{D}=1-\Sigma\left(\mathrm{Pi}_{\mathrm{i}}\right)^{2}$

- la réciprocité de l'indice de Simpson (1/D) qui mesure l'hétérogénéité de l'abondance des espèces ; elle a été calculée selon la formule: $1 / \mathrm{D}=1 / \Sigma(\mathrm{Pi})^{2}$;

- l'indice de Margalef (DMg) qui indique si la richesse spécifique est élevée ou non par la formule:
$\mathrm{DMg}=(\mathrm{S}-1) / \mathrm{LnN}$ où $\mathrm{S}$ est le nombre total d'espèces de l'échantillon et $\mathrm{N}$ le nombre total d'individus des espèces de de l'échantillon (MagurRan 2004).

Le taux de régénération (Tr) de chaque espèce a été évalué par la formule 2:

Formule 2: $\quad \operatorname{Tr}(\%)=\frac{\text { Nombre d'individus de diamètre }<5 \mathrm{~cm}}{\text { Nombre total d/individus dans } 1 / \mathrm{UAF}} \times 100$

Tous ces paramètres ont été calculés avec le tableur Excel.

À partir d'un seuil de $5 \mathrm{~cm}$, des classes de diamètre d'amplitude 5 ont été constituées afin d'établir la structure de Vitellaria paradoxa à l'aide d'histogrammes de distribution diamétrales. Une distribution théorique suivant la loi de Weibull a été réalisée à l'aide du logiciel MINITAB 14, pour caractériser la structure des populations de l'espèce. Le choix de la distribution de Weibull a été fait à la faveur de sa souplesse d'emploi et de la grande variabilité de formes de distribution qu'elle produit. La distribution de Weibull à trois paramètres (de position a, d'échelle ou taille b et de forme c) a été utilisée selon la formule 3:

Formule 3: $f(x)=\frac{c}{b}\left(\frac{x-a}{b}\right)^{c-1} \exp \left[-\left(\frac{x-a}{b}\right)^{c}\right]$

Le paramètre a correspond à la valeur seuil, c'est-à-dire à la plus petite valeur de diamètre retenue pour la constitution des histogrammes. Le paramètre $b$ est lié à la valeur centrale de la distribution des classes de diamètre. Le paramètre $\mathrm{c}$ traduit la structure observée et selon sa valeur détermine la forme de la distribution structurale des peuplements. Lorsque $\mathrm{c}<1$, la distribution est en "J inversé"; lorsque $\mathrm{c}=1$, la distribution est exponentielle négative. Si $1<\mathrm{c}<3$,6, la distribution est asymétrique positive. Pour $\mathrm{c}=3,6$, la distribution est approximativement normale et lorsque $\mathrm{c}>3,6$, la distribution est asymétrique négative.

\subsubsection{Analyses statistiques}

Les tests de Shapiro-Wilk et de Levene ont été réalisés pour tester respectivement la normalité et l'égalité des variances de chaque paramètre au sein des UAFs. Lorsque les hypothèses de normalité et d'égalité de variance étaient vérifiées, une analyse de variance à un facteur a été effectuée pour évaluer la différence de chaque paramètre entre les UAFs. Lorsque les moyennes sont significativement différentes et les effectifs suffisants, un test de comparaison multiple est appliqué; le test de Tukey (HSD: Honestly Significant Difference). Le logiciel R (R Development Core Team 2008) a permis d'effectuer ces tests statistiques.

\section{Resultats}

\subsection{Etats des perturbations anthropiques dans les UAFs étudiées}

L'ampleur et l'intensité des différents facteurs anthropiques à l'intérieur des UAFs sont rapportés dans la Fig. 2. Il ressort que toutes les UAFs parcourues présentent des traces de perturbations anthropiques dans des proportions variables. Le pâturage suivi par les feux non autorisé, ont été les facteurs anthropique les plus fréquents dans toutes les UAFs (plus de 60\% des placettes sont touchées) avec toutefois des 

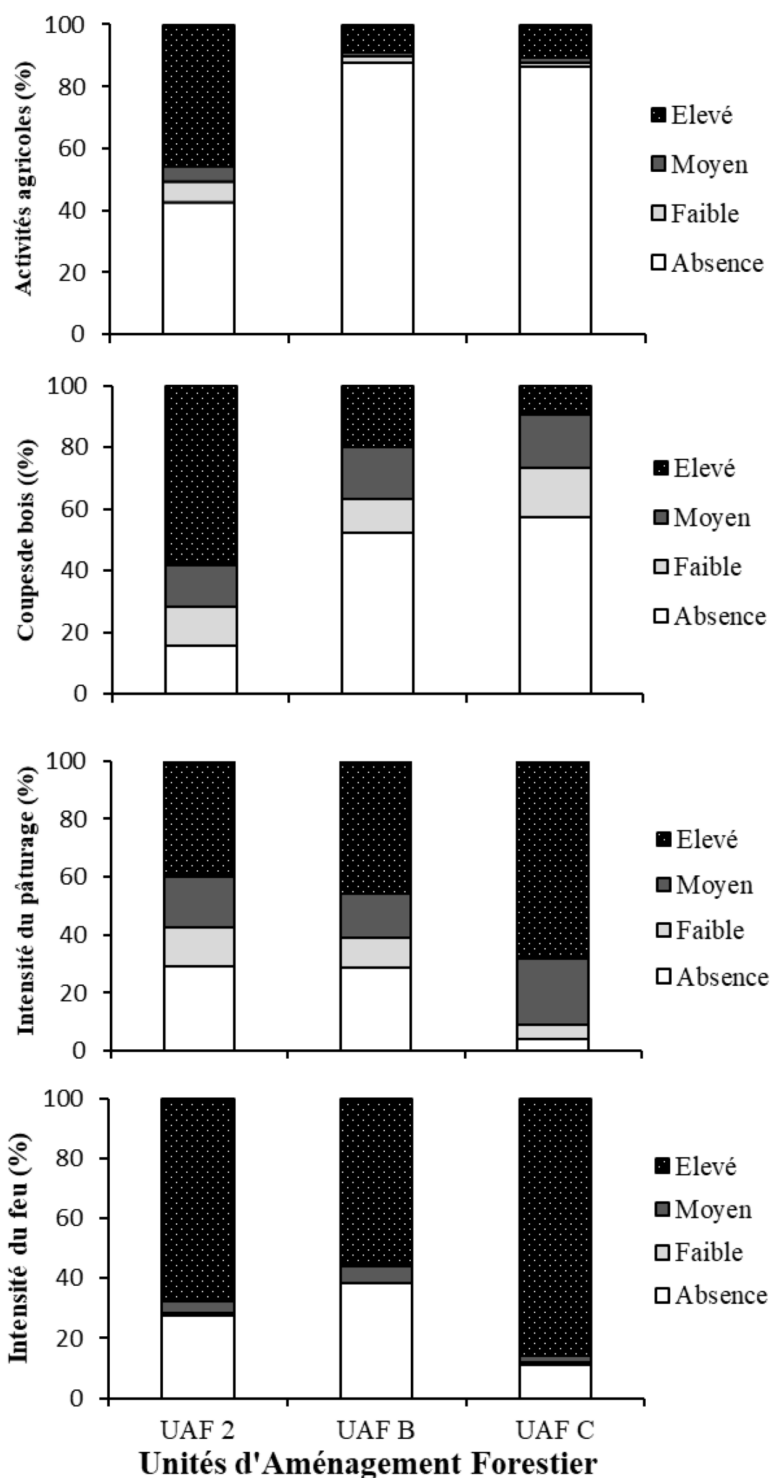

Fig. 2: Intensités des différents facteurs anthropiques en pourcentage (\%) de placettes affectées dans les UAFs étudiées / Intensities of the various anthropogenic factors in percentage $(\%)$ of plots affected in the studied FMUs.

incidences plus élevées dans l'UAF C (>90\%). Les coupes illégales de bois ont été observées également dans toutes les UAFs étudiées dans des proportions allant de $42 \%$ des placettes inventoriées (dans les UAFs C et B) à $84 \%$ dans l'UAF 2 qui a été, le plus touchée par ce facteur. Les traces d'activités agricoles ont été également plus présentes dans l'UAF 2 (57,5\%) par rapport aux deux autres UAFs $(<13 \%)$.

\subsection{Bilan floristique et caractéristiques structurales de la végétation des UAFs étudiées}

Le peuplement ligneux de la végétation des trois unités d'aménagement forestier (UAFs) comprend 96 espèces réparties en 67 genres et 26 familles. Les caractéristiques floristiques et dendrométriques des différentes UAFs sont résumées dans le Tableau 3. Le nombre de taxa a légèrement varié suivant les UAFs. A l'exception du nombre de familles qui a été plus élevé dans l'UAF C, l'UAF 2 a enregistré le plus grand nombre de taxa avec 86 espèces appartenant à 62 genres et 25 familles; les plus faibles nombre de taxa ont été notés dans les UAF B. Les indices de diversité mesurés ont été plus ou moins similaires dans les UAFs étudiées avec toutefois des valeurs plus élevées dans l'UAF 2 suivi respectivement par les UAFs C et B.

La nature et l'abondance des espèces a varié également suivant les UAFs (Tableau 4). Dans les UAFs 2 et B, Vitellaria paradoxa et Detarium microcarpum présentent les indices de valeur d'importance (IVI) les plus élevées tandis dans l'UAF C, il s'agit de Anogeissus leiocarpa suivi par Detarium microcarpum. La représentativité des familles à l'intérieur des UAFs (Tableau 5) révèle la domination de la famille des Fabaceae suivi de celle des Combretaceae et des Sapotaceae dans toutes les UAFs à l'exception de l'UAF $\mathrm{C}$ où les Combretaceae ont présenté la plus grande valeur d'importance des familles (FIV).

Les caractéristiques structurales du peuplement ligneux montrent des valeurs de densité et de surface terrière très variables suivant les différentes UAFs (Tableau 3). La densité des ligneux varie significativement suivant les UAFs ( $\mathrm{df}=2$ $; F=27,08 ; p<0,0001)$ avec des valeurs plus élevées dans les UAFs $\mathrm{C}$ et $\mathrm{B}$ par rapport à UAF 2. La surface terrière des espèces a montré également des différences significatives suivant les UAFs $(\mathrm{df}=2 ; \mathrm{F}=28,6 ; \mathrm{p}<0,0001)$ avec des valeurs plus élevées dans l'UAF C $\left(67,1 \mathrm{~m}^{2} / \mathrm{ha}\right)$ suivies respectivement par celles de l'UAF B et de l'UAF 2 qui a enregistré la plus faible surface terrière.

Les résultats relatifs au potentiel de régénération des espèces sont reportés dans le Tableau 6 . Le taux de régénération est relativement plus élevé dans l'UAF $2(49,75 \%)$ suivi par celui de l'UAF B $(38,89 \%)$ et faible dans l'UAF

Tableau 3: Paramètres floristiques et dendrométriques de la végétation des trois UAFs / Floristic and dendrometric parameters of the vegetation in the three FMUs.

\begin{tabular}{|l|l|l|l|}
\hline Paramètres & UAF 2 & UAF B & UAF C \\
\hline Familles (nombre total) & 24 & 21 & 25 \\
\hline Genres (nombre total) & 62 & 53 & 61 \\
\hline Nombre total d'espèces & 86 & 73 & 81 \\
\hline Indice de Margalef (DMg) & 8,05 & 7,30 & 8,03 \\
\hline Indice de diversité de Shannon-Weaver (H') & 3,26 & 2,92 & 3,01 \\
\hline Réciproque de l'indice de Simpson (1/D) & 17,85 & 9,73 & 12,62 \\
\hline Indice d'équitabilté de Simpson (D) & 0,94 & 0,90 & 0,92 \\
\hline Densité (N/ha) & $382 \pm 61 \mathrm{a}$ & $690 \pm 89 \mathrm{~b}$ & $718 \pm 55 \mathrm{~b}$ \\
\hline Surface terrière $\left(\mathrm{m}^{2} / \mathrm{ha}\right)$ & $40,7 \pm 12,1 \mathrm{a}$ & $47,2 \pm 10,4 \mathrm{a}$ & $67,1 \pm 15,8 \mathrm{~b}$ \\
\hline
\end{tabular}


Tableau 4: Les cinq espèces les plus abondantes dans chaque UAF selon l'ordre décroissant de leur Indice de Valeur d'importance/ the five most abundant species in each FMU in descending order of their Importance Value Index.

\begin{tabular}{|c|c|c|c|c|c|}
\hline UAFs & Espèces & Den. rel. (\%) & Dom. rel. (\%) & Frep. Rel. (\%) & IVI (\%) \\
\hline \multirow{7}{*}{ UAF 2} & Vitellaria paradoxa & 6,56 & 26,24 & 4,81 & 37,61 \\
\hline & Detarium microcarpum & 13,79 & 4,04 & 4,53 & 22,37 \\
\hline & Anogeissus leiocarpa & 6,73 & 9,43 & 3,21 & 19,36 \\
\hline & Piliostigma thonningii & 8,47 & 2,86 & 4,03 & 15,36 \\
\hline & Lannea acida & 0,72 & 7,93 & 3,11 & 11,77 \\
\hline & Total & 36,28 & 50,50 & 19,69 & 106,46 \\
\hline & Autres & 63,72 & 49,50 & 80,31 & 193,54 \\
\hline \multirow{7}{*}{ UAF B } & Detarium microcarpum & 27,12 & 11,17 & 5,10 & 43,39 \\
\hline & Vitellaria paradoxa & 7,52 & 16,89 & 4,81 & 29,22 \\
\hline & Lannea acida & 0,93 & 12,07 & 4,17 & 17,17 \\
\hline & Anogeissus leiocarpa & 4,49 & 8,80 & 3,42 & 16,71 \\
\hline & Burkea africana & 1,14 & 10,57 & 3,71 & 15,41 \\
\hline & Total & 41,21 & 59,49 & 21,21 & 121,91 \\
\hline & Autres & 58,79 & 40,51 & 78,79 & 178,09 \\
\hline \multirow{7}{*}{ UAF C } & Anogeissus leiocarpa & 15,88 & 30,18 & 4,79 & 50,86 \\
\hline & Detarium microcarpum & 16,09 & 6,13 & 4,43 & 26,66 \\
\hline & Vitellaria paradoxa & 6,60 & 14,75 & 4,72 & 26,07 \\
\hline & Combretum nigricans & 7,53 & 2,42 & 3,65 & 13,60 \\
\hline & Combretum molle & 2,87 & 5,53 & 4,22 & 12,62 \\
\hline & Total & 48,98 & 59,01 & 21,82 & 129,81 \\
\hline & Autres & 51,02 & 40,99 & 78,18 & 170,19 \\
\hline
\end{tabular}

IVI = Indice de Valeur d'Importance

C (28,87\%). Detarium microcarpum a enregistré le meilleur taux de régénération dans toutes les UAFs; elle est suivie par Pteleopsis suberosa dans les UAFs 2 et B, par Anogeissus leiocarpa dans l'UAF C. En considérant les espèces les plus fréquentes et abondantes dans les classes adultes, il ressort que Anogeissus leiocarpa et Burkea africana présentent des faibles taux de régénération dans les UAFs 2 et B (TR $<2 \%$ ) tout comme Combretum molle dans l'UAF C; ce qui pose le problème de renouvèlement des peuplements de ces espèces.

\subsection{Etats des peuplements de Vitellaria paradoxa dans les UAFs étudiées}

\subsubsection{Structures démographiques et capacité de régéné- ration}

Les caractéristiques structurales des peuplements de $\mathrm{Vi}$ tellaria paradoxa varient significativement suivant les différentes UAFs (Tableau 7). La densité du peuplement de l'espèce varie significativement suivant les UAFs $(\mathrm{df}=2$ ; $\mathrm{F}=27,8 ; \mathrm{p}=0,04)$ avec des valeurs plus élevées dans l'UAF C et dans l'UAF B par rapport à celles de l'UAF 2.

Tableau 5: Les trois familles les plus représentées dans chaque UAF selon l'ordre décroissant de leur FIV (Valeur d'Importance d'une Famille) / The three most represented families in each FMU in descending order of their FIV (Family Importance Value).

\begin{tabular}{|c|c|c|c|c|c|}
\hline UAFs & Familles & Dens. rel. (\%) & Dom. rel. (\%) & Div. rel. (\%) & FIV (\%) \\
\hline \multirow{5}{*}{ UAF 2} & Fabaceae & 40,58 & 31,35 & 33,72 & 105,65 \\
\hline & Combretaceae & 30,26 & 19,91 & 11,63 & 61,80 \\
\hline & Sapotaceae & 6,56 & 26,24 & 1,16 & 33,96 \\
\hline & Total & 77,40 & 77,50 & 46,51 & 201,41 \\
\hline & Autres & 22,60 & 22,50 & 53,49 & 98,59 \\
\hline \multirow{5}{*}{ UAF B } & Fabaceae & 47,43 & 37,03 & 36,84 & 121,30 \\
\hline & Combretaceae & 28,17 & 21,66 & 13,16 & 63,00 \\
\hline & Sapotaceae & 7,52 & 16,89 & 1,32 & 25,73 \\
\hline & Total & 83,12 & 75,59 & 51,32 & 210,03 \\
\hline & Autres & 16,88 & 24,41 & 48,68 & 89,97 \\
\hline \multirow{5}{*}{ UAF C } & Combretaceae & 40,13 & 42,38 & 12,35 & 94,85 \\
\hline & Fabaceae & 36,82 & 24,99 & 29,63 & 91,44 \\
\hline & Sapotaceae & 6,60 & 14,75 & 1,23 & 22,58 \\
\hline & Total & 83,55 & 82,12 & 43,21 & 208,88 \\
\hline & Autres & 16,45 & 17,88 & 56,79 & 91,12 \\
\hline
\end{tabular}


Tableau 6: Taux de régénération (TR) dans les différentes UAFs avec les cinq espèces présentant les meilleurs taux Regeneration rate in the different FMUs with the five most represented species.

\begin{tabular}{|c|c|c|}
\hline UAFs & Espèces & TR (\%) \\
\hline \multirow{7}{*}{ UAF 2} & Detarium microcarpum & 5,76 \\
\hline & Pteleopsis suberosa & 5,49 \\
\hline & Dichrostachys cinerea & 4,03 \\
\hline & Piliostigma thonningii & 3,95 \\
\hline & Vitellaria paradoxa & 3,06 \\
\hline & Autres & 27,46 \\
\hline & Total & 49,75 \\
\hline \multirow{7}{*}{ UAF B } & Detarium microcarpum & 8,76 \\
\hline & Pteleopsis suberosa & 5,17 \\
\hline & Vitellaria paradoxa & 3,07 \\
\hline & Combretum glutinosum & 2,14 \\
\hline & Piliostigma thonningii & 1,93 \\
\hline & Autres & 17,81 \\
\hline & Total & 38,89 \\
\hline \multirow{7}{*}{ UAF C } & Detarium microcarpum & 5,03 \\
\hline & Anogeissus leiocarpa & 3,99 \\
\hline & Combretum nigricans & 3,60 \\
\hline & Vitellaria paradoxa & 1,53 \\
\hline & Pteleopsis suberosa & 1,49 \\
\hline & Autres & 13,22 \\
\hline & Total & 28,87 \\
\hline
\end{tabular}

La surface terrière de Vitellaria paradoxa a montré également des différences significatives suivant les UAFs $(\mathrm{df}=$ $2 ; \mathrm{F}=3,303 ; \mathrm{p}=0,03$ ) avec cette fois des valeurs plus élevées dans les UAFs 2 et $\mathrm{C}\left(12,1 \mathrm{~m}^{2} / \mathrm{ha} ; 11,4 \mathrm{~m}^{2} /\right.$ ha respectivement) par rapport à l'UAF $\mathrm{B}\left(8,7 \mathrm{~m}^{2} / \mathrm{ha}\right)$. Les résultats relatifs au potentiel de régénération de l'espèce indiquent de faibles taux de régénération sur la totalité des UAFs étudiées (autour de 3\%) avec toutefois des valeurs plus faibles dans l'UAF C. Quant aux résultats sur les diamètres moyens des individus, il ressort que l'UAF 2 présente les plus gros individus suivi de ceux de l'UAF B, pendant que les plus faibles diamètres sont observés dans l'UAF C.

La distribution des classes de diamètre et de hauteur des peuplements de Vitellaria paradoxa dans les différents UAFs est présentée dans la Fig. 3. L'examen de ces distributions conformément à la loi de Weibull montre des structures diamétrales en forme de $\mathrm{L}$ ou de $\mathrm{J}$ inversé dans les toutes les trois UAFs investiguées (les valeurs du paramètre de forme $\mathrm{c}$ sont toutes inférieures à $1(0,64 \leq \mathrm{c} \leq 0,76)$; ce qui indique une importance majeure d'individus de faibles diamètres (Fig. 3A). L'analyse des structures en hauteur montre pratiquement les mêmes allures que la distribution des classes de diamètre dans les UAFs sauf que les valeurs du paramètre de forme $\mathrm{c}$ différent légèrement (Fig. 3B). En effet, le paramètre de forme $\mathrm{c}$ est inférieur à 1 seulement dans l'UAF $2(\mathrm{c}=0,089)$ tandis que sa valeur est d'environ 1 dans les UAFs $B$ et $C$, indiquant ainsi une forte présence de jeunes pieds mais également d'individus dont le développement pourrait être compromis par les strates arborescentes, souvent qualifiés de rabougris.
Tableau 7: Paramètres dendrométriques et taux de régénération de Vitellaria paradoxa dans les différentes UAFs / Dendrometric parameters and regeneration rate of Vitellaria paradoxa in the different FMUs.

\begin{tabular}{|l|l|l|l|l|}
\hline UAFs & $\begin{array}{l}\text { Densité } \\
\mathbf{( N / h a )}\end{array}$ & $\begin{array}{l}\text { Surface ter- } \\
\text { rière }\left(\mathbf{m}^{\mathbf{2}} / \mathbf{h a}\right)\end{array}$ & $\begin{array}{l}\text { Diamètre } \\
\text { moyen } \mathbf{( c m})\end{array}$ & $\begin{array}{l}\text { Taux de ré- } \\
\text { génération } \\
\mathbf{( \% )}\end{array}$ \\
\hline UAF 2 & $62 \pm 5 \mathrm{a}$ & $12,1 \pm 4,3 \mathrm{~b}$ & $39,60 \pm 10,17$ & 3,06 \\
\hline UAF B & $82 \pm 8 \mathrm{~b}$ & $8,7 \pm 2,4 \mathrm{a}$ & $29,36 \pm 9,92$ & 3,07 \\
\hline UAF C & $89 \pm 9 \mathrm{~b}$ & $11,4 \pm 5,8 \mathrm{~b}$ & $23,54 \pm 12,72$ & 1,53 \\
\hline
\end{tabular}

\subsubsection{Etat sanitaire des peuplements de Vitellaria para-} doxa

La Fig. 4 résume la situation sanitaire des peuplements de Vitellaria paradoxa dans les UAFs investiguées. De l'analyse de cette figure, il ressort que les peuplements de l'espèce présente une situation sanitaire satisfaisante car la proportion des individus sans défaut visible est supérieure à $85 \%$ sur l'ensemble des UAFs. Cependant, les problèmes sanitaires les plus récurrents sont respectivement la présence des individus attaqués par des parasites de Loranthaceae, des individus semi-morts ou à cimes plus ou moins desséchées et des individus ébranchés. Aussi, la situation sanitaire de l'espèce varie d'une unité à une autre suivant l'incidence des agents ou facteurs causaux. Bien qu'elle ait des proportions en individus ébranchés et semi-morts plus élevés (3\%), l'UAF C apparait comme présentant la meilleure situation sanitaire (91\% d'individus sans défaut visible) suivi respectivement par l'UAF B et l'UAF 2 à l'intérieur desquelles, les peuplements de l'espèce souffrent surtout de l'infestation des parasites de Loranthaceae ( $>6 \%$ ).

\section{Discussion}

\subsection{Ampleur des facteurs de perturbation anthropiques affectant les UAFs}

Les résultats indiquent que toutes les UAFs présentent des traces de perturbations anthropiques dans des proportions élevées. Le pâturage suivi par les feux non autorisé ont été les facteurs anthropique les plus fréquents dans toutes les UAFs (plus de $60 \%$ des placettes sont touchées) avec toutefois des incidences plus élevées dans l'UAF C (>90\%) qui est l'unité d'aménagement la plus éloignée de l'unité de gestion du CAF. Les incidences élevées du feu et du pâturage dans les UAFs révèlent donc des failles dans le dispositif de surveillance mis en place. Ces résultats corroborent ceux obtenus par SAWADOGo (2009) qui a indiqué que les prescriptions prévues dans le cahier de charge, en matière d'administration des feux précoces et d'interdiction du pâturage, sont rarement respectées du fait du manque de suivi et de surveillance des parcelles. Les incidences élevées de ces deux facteurs dans l'UAF C par rapport aux deux autres, pourrait donc s'expliquer par sa situation très éloignée de l'unité de gestion du CAF. En effet, des investigations conduites par Yoni \& Sedogo (2015) dans les CAF $\mathrm{du}$ Centre- Ouest, indiquent que le contrôle du service technique et forestier dans le CAF de Cassou est insuffisant. En plus, la proximité des villages riverains, constituent une opportunité de ravitaillement en ressource fourragère par le bétail airant ou les pasteurs transhumants. Les coupes illégales de bois et les traces d'activités agricoles, ont été quant à elles plus présentes dans l'UAF 2 malgré 
(A)
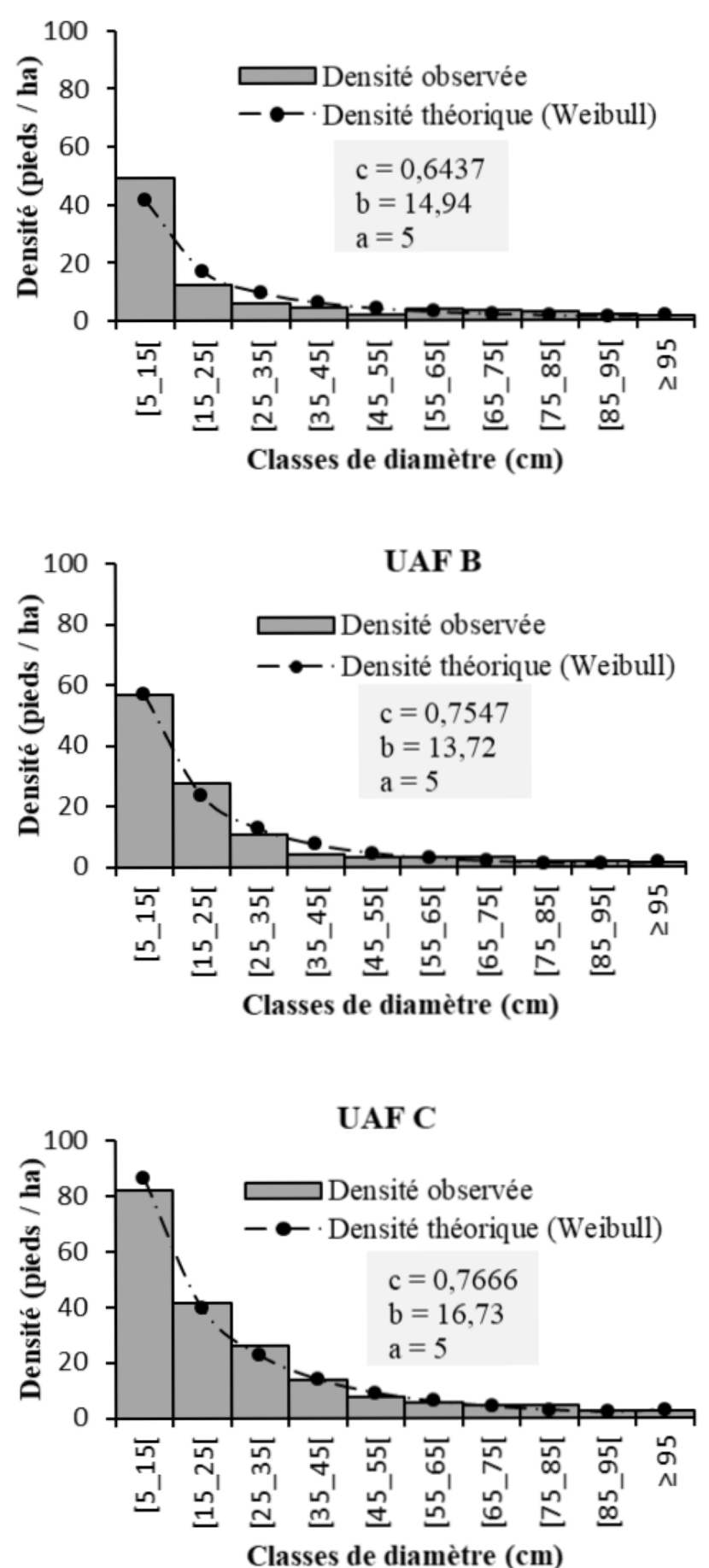

(B)

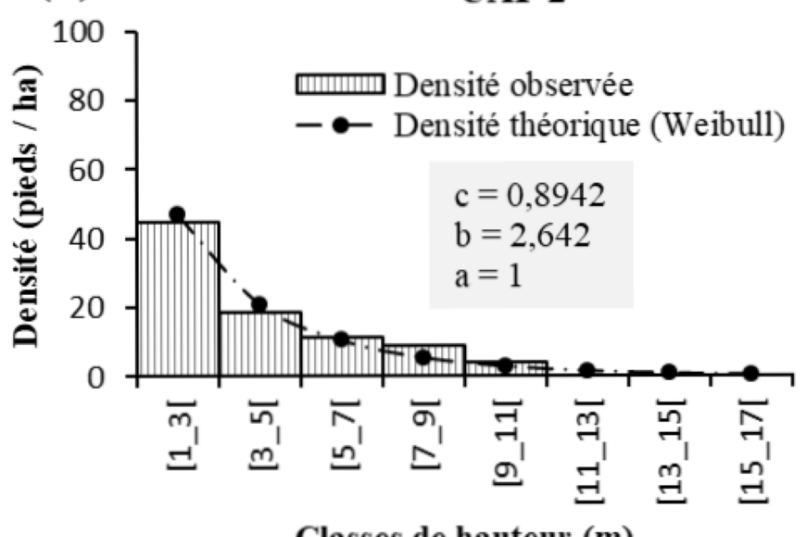

Classes de hauteur (m)

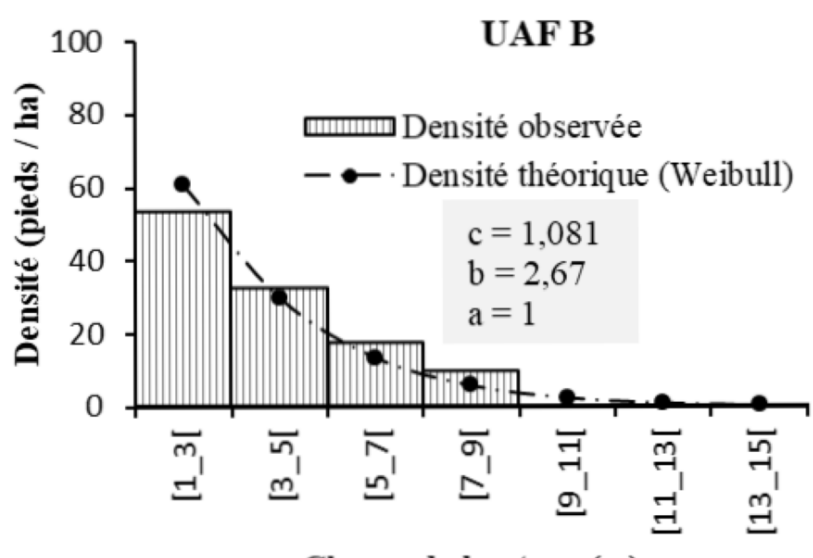

Classes de hauteur (m)

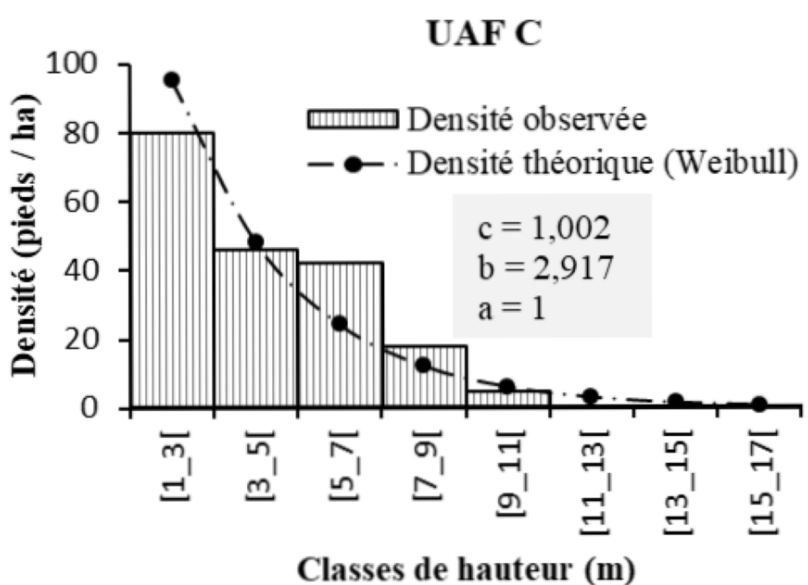

Fig. 3: Structure en diamètre (A) et en hauteur (B) des peuplements de Vitellaria paradoxa dans chaque UAF avec imposition de leurs distributions suivant la loi de Weibull à trois paramètres (de position $\mathrm{a}$, d'échelle b et de forme $\mathrm{c}$ ) / Diameter (A) and height (B) structure of Vitellaria paradoxa in each FMU with imposition of their distribution according to the three-parameter Weibull $(\mathrm{a}=$ thresh, $\mathrm{b}=\mathrm{scale}$ and $\mathrm{c}$ = shape).

sa relative proximité de l'unité de gestion du CAF. Ces résultats s'expliquent par la forte pression anthropique entretenue par les populations des nombreux villages riverains, à travers dans la recherche de bois de chauffe et la pratique d'une agriculture extensive.

\subsection{Diversité floristique et dynamique des ligneux}

La présente étude révèle que la flore ligneuse des trois UAFs du Chantier d'Aménagement Forestier (CAF de Cassou, est riche de quatre-vingt-seize (96) espèces ligneuses réparties en 67 genres et 26 familles dominées par la famille des Fabaceae. Comparée aux résultats de Boussim \& al. (2009) qui ont recensés soixante-huit (68) espèces dans les unités d'aménagement forestier du même chantier d'aménagement (Cassou), la flore récensée est plutôt riche. Cette richesse floristique est également plus importante que celles enregistrées dans le CAF de Tiogo (88 espèces ligneuses) par SAVADOGO \& al. (2002) et dans le CAF du Nazinon (90 espèces ligneuses) par SAWADOGO (2007) ; toutefois elle reste faible 


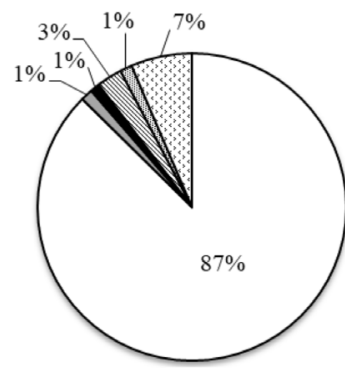

Légende

口Sans défaut visible

口Ebranchés

Brûlés

๑ Semi-morts ou cime desséchées

由Morts sur pied

ĐParasités

UAF 2

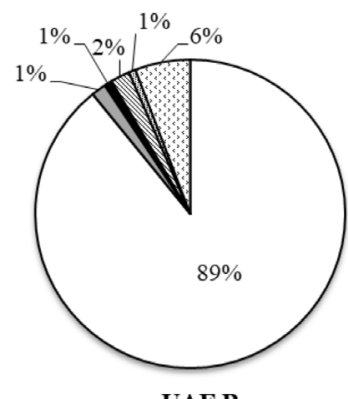

UAF B

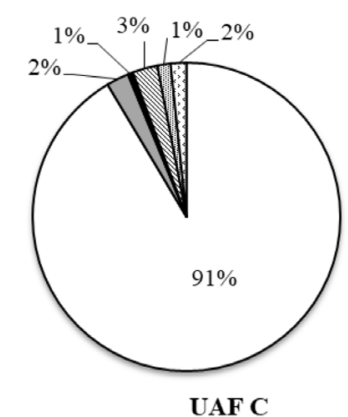

Fig. 4: Etat sanitaire des peuplements de Vitellaria paradoxa dans chacune des UAFs étudiées / Health status of Vitellaria paradoxa stands in each studied FMUs.

comparée à celle enregistrée dans le domaine soudanien du Togo avec 111 espèces d'arbres réparties en 79 genres et 32 familles (Dourma \& al. 2012). Plusieurs facteurs peuvent expliquer ces différences, dont notamment les variations des conditions pédoclimatiques et leurs influences sur la distribution spatiale et géographique des flores (BONDE \& al. 2013).

La similitude de la composition floristique de la strate ligneuse dans les différentes UAFs, seraient liée au fait qu'elles soient toutes dans la même zone phytogéographique, subissant les mêmes conditions climatiques et le même système d'aménagement et à des degrés près les mêmes pressions anthropiques. Aussi, dans toutes les UAFs, les indices de diversité mesurés indiquent qu'il n'y a pas de différence significative d'une UAF à l'autre. Ces résultats sont en accords avec ceux de Kengne \& al. (2018), qui indiquent que la périodicité des activités d'exploitation dans les UAFs, fait que les phases de perturbation n'ont pas de grandes influences sur la diversité de la flore et la richesse spécifique. En effet, les coupes frauduleuses du bois vert pour les besoins tels que la construction, l'énergie et l'exploitation des espèces ligneuses comme fourrage portent sur des espèces précises, si bien que ces activités illégales dans les UAFs, ne sont actuellement pas assez importantes pour provoquer une baisse conséquente de la diversité floristique. Néanmoins, ces facteurs de perturbations agissent négativement sur la structure des ligneux (BONDE et al. 2013) car les défrichements combinés à l'exploitation presque abusive de certaines espèces conduisent à la réduction de leurs paramètres dendrométriques, surtout dans les zones anthropisées ; cela expliquerait les faibles densités en ligneux dans l'UAF 2, plus perturbé par les coupes illégales de bois par rapport aux deux autres UAFs. L'une des principales causes de régression des formations ligneuses est la raréfaction des jeunes individus susceptibles de remplacer les sujets vieillissants (OUEDRAOGO \& al. 2006). Les résultats de la présente étude indiquent que le taux de régénération avec une prédominance de Detarium microcarpum est relativement plus élevé dans l'UAF 2 suivi par l'UAF $\mathrm{B}$ et faible dans l'UAF $\mathrm{C}$ où les incidences du pâturage et des feux incontrôlés étaient plus élevées. Selon SAwADOGo (2009), le faible taux de régénération des ligneux est la conséquence du surpâturage ainsi que des feux de brousses répétés et incontrôlés qui ne laissent pas suffisamment de temps aux espèces pour se reconstituer, justifiant ainsi nos résultats. Les faibles taux de régénération de certaines espèces fréquentes et abondantes dans les classes adultes, comme Anogeissus leiocarpa et Burkea africana présentent dans les UAFs 2 et B et Combretum molle dans l'UAF C, seraient la conséquence de l'incidence des facteurs de perturbation. Le surpâturage entraîne la réduction de la régénération naturelle des ligneux (JURISCH \& al. 2016), la baisse de la couverture herbacée, la mise à nue du sol et son endurcissement (Kessler \& GeERLing 2006). Des observations analogues ont été faites également par SAwADOGo (2007) et Boussim \& al. (2009), qui ont constaté que ces espèces étaient menacées de disparition à cause de leur régénération quasi nulle. La coupe abusive de bois a déjà été relevée comme un facteur important dans la dynamique régressive des ligneux (BAGGNian \& al. 2013). La bonne régénération de Detarium microcarpum corrobore les observations de Kabore (2004) et de SAwAdogo (2007) dans d'autres CAF du Burkina Faso, et s'explique selon Bellefontaine \& al. (2000) par les grandes capacités de drageonnage de l'espèce qui constituent un atout de bonne régénération car les drageons ont une croissance plus rapide que les semis.

\section{3 Etats des peuplements de Vitellaria paradoxa dans les UAFs étudiées}

La distribution des peuplements de Vitellaria paradoxa sur l'ensemble des UAFs, présente des paramètres de formes indiquant des structures en « L ». Selon Dan Guimbo \& al. (2010), les individus jeunes assurent l'avenir de la formation naturelle tandis que ceux de gros arbres résultant de la sélection naturelle sont des semenciers qui assurent la pérennité du peuplement à travers la production des graines. La prédominance d'individus de faible diamètre et de hauteur dans les différentes UAFs assurent ainsi une dynamique structurale stable. L'analyse des caractéristiques dendrométriques montre des variations significatives suivant les UAFs avec des valeurs de densité plus élevées dans l'UAF $\mathrm{C}$ et de surface terrière plus élevées dans les UAFs 2 et $\mathrm{C}$. Cette différence de répartition et d'abondance de l'espèce dans les UAFs s'explique par les facteurs écologiques et les pressions anthropiques qui s'exercent sur elle. La densité élevée observée au niveau de l'UAF C, s'explique par les conditions stationnelles et écologique favorable de cette UAF avec une végétation généralement plus dense par rapport aux autres UAFs. Les densités observées dans le cadre de la présente étude sont similaires à celles obtenues dans la même zone (Boussim \& al. 2009) mais nettement moins élevées que celles obtenues à Saponé (222 pieds /ha) par RABIOU \& al. (2016). Cette différence s'explique par l'intervention d'un projet d'aménagement qui dans le cadre de ces activités de restauration du paysage a réalisé des plantations de l'espèce dans ce milieu. Toutefois ces densités sont nettement supérieures à celles observées par BoFFA (2000) au Mali, dans les agrosystèmes ( 2 pieds / ha en 
secteur nord soudanien, 19 pieds /ha en secteur sud soudanien et 52 pieds /ha en secteur soudano-guinéen). Les fortes surfaces terrières observées au niveau de l'UAF 2 malgré sa faible densité, s'explique par le faite que l'importance de la surface terrière est corrélée à la présence et à la fréquence des individus à gros tronc qui sont plus représentée dans cette UAF ; cependant les fortes surfaces terrières observées au niveau de l'UAF C s'explique par la forte densité de l'espèce observée dans son sein, mais dont les individus sont caractérisés par des diamètres moyens et hauteur moyenne faible (Tableau 7).

Les résultats relatifs au potentiel de régénération de l'espèce indiquent de faibles taux de régénération sur la totalité des UAFs étudiées avec toutefois des valeurs plus faibles dans l'UAF C. Ces résultats contrastent avec ceux obtenus par RABIOU \& al. (2016) qui ont constaté une forte densité de régénération dans la forêt de Saponé en zone Nord-soudanienne. Les faibles taux de régénération de l'espèce dans les UAFs en général et dans l'UAF C, en particulier, peuvent s'expliquer par l'effet des facteurs de dégradation tels le surpâturage et les feux de brousses incontrôlés (JURISCH \& al. 2016). En effet, plusieurs des plantules qui émergent sont éliminées soit par les effets de la sècheresse en saison sèche, soit le feu de végétation ou par les dents des herbivores qui parcourent chaque jour ces UAFs. Cette remarque rejoint les observations de BOKARY \& al. (2004) qui expliquent ces difficultés d'affranchissement des individus juvéniles de $\mathrm{V} i$ tellaria paradoxa par les effets néfastes des feux de brousse qui ne favorisent pas l'évolution des plantules qui apparaissent. Par ailleurs, les feux précoces ou d'aménagement peuvent aussi entraîner une mortalité très élevée jeunes individus de l'ensemble des espèces.

L'état sanitaire des peuplements de Vitellaria parado$x a$ dans les UAFs est satisfaisant avec un taux d'individus sans défaut visible supérieur à $85 \%$ et surtout dans l'UAF C $(91 \%)$. Ces résultats sont en conformité avec ceux obtenus par SAWADOGo (2007) qui a également indiqué que l'espèce était victime de pratiques contrevenantes au plan d'aménagement et au cahier de charge, notamment la coupe pour le bois de feu. Toutefois, il est ressorti dans la présente étude que la principale menace sanitaire de l'espèce dans les UAFs est l'attaque des parasites de la famille des Loranthaceae avec des incidences plus élevées dans l'UAF 2 suivi de l'UAF B et faible dans l'UAF C. Cette différence des taux d'infestation constater dans les UAFs peut être liée à la fréquence et la taille des arbres, car selon Koffi \& al. (2014), le taux de parasitage des Loranthaceae est plus élevé chez les gros arbres par rapport aux arbustes. En effet, selon Boussim (2002), la dissémination des Loranthaceae se fait par des oiseaux qui se nourrissent de la viscine (pulpe collante) de leurs fruits. Ces oiseaux auraient donc une préférence des gros arbres dont l'ombrage procure plus de sécurité que les arbustes. Le faible taux de parasitage obtenu dans l'UAF C pourrait s'expliquer par le type de végétation caractérisé par un nombre moins élevé d'arbres défavorisant ainsi la dissémination des Loranthaceae.

\section{ConClusion}

Les investigations sur la végétation des trois (3) unités d'aménagement forestiers (UAFs) du CAF de Cassou a permis de mettre en évidence les diverses perturbations anthropiques exercées sur la végétation, d'évaluer la diversité floristique des ligneux et de décrire la structure ainsi que l'état sanitaire des peuplements de Vitellaria paradoxa. Il ressort que:

(i) l'exploitation frauduleuse et excessive du bois, couplée à la pression agricole et pastorale, compromet la pérennité de ces UAFs. Le pâturage suivi par les feux non autorisé ont été les facteurs de perturbations anthropiques les plus fréquents dans toutes les UAFs avec toutefois des incidences plus élevées dans l'UAF C (>90\%). Les coupes illégales de bois et les activités agricoles ont été plus manifestent dans l'UAF 2;

(i) les indices de diversité mesurés ont été plus ou moins similaires dans toutes les UAFs étudiées avec toutefois des valeurs plus élevées dans l'UAF 2 suivi respectivement par les UAFs C et B;

(iii) les caractéristiques dendrométriques des peuplements de Vitellaria paradoxa diffèrent suivant les UAFs avec des valeurs de densité et de surface terrière plus élevées dans l'UAF C tandis que la régénération est plus importante dans l'UAF 2 et faible dans l'UAF C. Toutefois, les allures des structures en diamètre et en hauteur de l'espèce montrent que les individus jeunes sont les plus représentés dans toutes les UAFs;

(iv) la situation sanitaire de Vitellaria paradoxa est satisfaisante sur l'ensemble des UAFs, même si la principale menace est l'attaque des parasites de Loranthaceae avec un taux infestation plus élevé dans l'UAF 2 suivi respectivement par l'UAF B et l'UAF C.

Partant des hypothèses de départ, les résultats obtenus montrent que les UAFs proches de l'unité de gestion ont été moins perturbées par une partie des facteurs notamment le pâturage et les feux incontrôlés; ce qui confirme partiellement la première hypothèse. Cependant, la deuxième hypothèse est rejetée car la structure démographique des peuplements de Vitellaria paradoxa n'a été fonction du gradient d'anthropisation définit en fonction de la proximité de l'unité de gestion.

\section{REMERCIEMENTS}

Les auteurs remercient le Center for International Forestry Research (CIFOR) qui a commandité cette étude. Les remerciements s'adressent particulièrement à Mr Michael Philippe Bessike Balinga, pour sa collaboration et son assistance logistique ainsi qu'à $\mathrm{Mr}$ Abdoulaye Rabdo pour son appui dans la réalisation des cartes.

\section{REFERENCES}

Baggnian I, Adamou M, Adam T \& Mahamane A (2013): Impact des modes de gestion de la Régénération Naturelle Assistée des ligneux (RNA) sur la résilience des écosystèmes dans le Centre-Sud du Niger. Journal of Applied Biosciences 71: $5742-5752$.

Belem M, Zoungrana M \& Nabaloum M (2018): Les effets combinés du climat et des pressions anthropiques sur la 
forêt classée de Toéssin, Burkina Faso. Int. J. Biol. Chem. Sci. 12(5): 2186-2201.

Bellefontaine R, Edelin C, Ichaou A, Du Laurens D, MONSARRAT A \& LOQUAI C (2000): Le drageonnage, alternative aux semis et aux plantations de ligneux dans les zones semi-arides: protocole de recherches. Sécheresse, 11 (4): 221-226.

BofFA JM (2000): Les parcs agroforestiers en Afrique Subsaharienne. Cahier FAO cas du maïs (Zea mays L.) Associé au Karité (Vitallaria paradoxa) dans la Zone de Conservation, Cahier FAO, guide de conservation 34, FAO, Rome : $1-251$.

Bokary AK, Bouvet JM \& Picard N (2004): Size class distribution and spatial pattern of Vitellaria paradoxa in relation to farmer practices in Mali. Agroforestry Systems, 60: 3-11.

Bonde L, Ouedraogo O, Kagambega WF \& Boussim IJ (2013). Impact des gradients topographique et anthropique sur la diversité des formations ligneuses soudaniennes. Bois et Forêts des Tropiques 318 (4): 15 - 25.

Boussim IJ (2002): Les Phanérogames parasites du Burkina Faso : inventaire, taxonomie, écologie, et quelques aspects de leur biologie. Cas particulier des Loranthaceae parasites du karité. Thèse de doctorat d'état ès Sciences naturelles. 285 p.

Boussim IJ, Ouedraogo A \& Lankoande B (2009): Etude des impacts écologiques dans les unités d'aménagements forestiers des régions du Centre-Nord et du centre-Ouest. In: Kabré AM, Somda J, Savadogo M, Nianogo AJ (eds). Bois-énergie au Burkina Faso: Consolidation des moyens d'existence durable (2006 - 2009), Ouagadougou, Burkina Faso: Bureau UICN - Burkina Faso : 115-139.

Coulibaly-Lingani P, Savadogo P, Tigabu M \& Oden PC (2011): Factors influencing people's participation in the forest management program in Burkina Faso, West Africa. Forest Policy and Economics 13 (4): 292-302.

CurTis JT \& Mcintosh RP (1950): The interrelations of certain analytic and synthetic phytosociological characters. Ecology 31:434-455.

DAJOz R (2008): La biodiversité: l'avenir de la planète et de l'homme. Ellipses, Paris. 275 p.

Dan Guimbo I, Mahamane A \& Ambouta KJM (2010): Peuplement des parcs à Neocarya macrophylla (Sabine) Prance et à Vitellaria paradoxa (Gaertn. C.F.) dans le sudouest nigérien: diversité, structure et régénération. Int. J. Biol. Chem. Sci., 5, (4): 1706 - 1720.

Dourma M, Wala K, Guelly KA, Bellefontaine R, Deleporte P, Akpavi S, Batawila K \& Akpagana K (2012): Typologie, caractéristiques structurales et dynamique des faciès forestiers fragiles à Isoberlinia spp. en vue de leur gestion au Togo. Bois et Forêts des Tropiques, 313 (3) : 20-33

Etongo D, Kanninen M, Epule Epule T \& Fobissie K (2018): Assessing the effectiveness of joint forest management in Southern Burkina Faso: A SWOT-AHP analysis. Forest Policy and Economics 90: 31-38, https://oi. org/10.1016/j.forpol.2018.01.008

FAO (2003): Gestion de la fertilité des sols pour la sécurité alimentaire en Afrique subsaharienne. Rome, Italie, 63 p.

FAO (2015): Global forest resources assessment 2015. Food and Agriculture Organization of the United Nations, Rome, $253 \mathrm{p}$.
JuRISCH K, BERnhaRdT-RÖMERMANN M, WitTIG R \& HAHN $K$ (2016): Impact of land use on juvenile densities of woody plants in a West African savanna. Flora et Vegetatio SudanoSambesica 19, 18-34

KABORE C (2004): Référentiel technique d'aménagement des forêts au Burkina Faso, BKF/007PAFDK, Ouagadougou, 133p.

Kengne OC, Zapfack L, Garcia C, Noiha NV \& NkongMENECK B-A (2018): Diversité floristique et structurale de deux forêts communautaires sous exploitation au $\mathrm{Ca}$ meroun: cas de Kompia et Nkolenyeng. European scientific journal (14) $24: 245-271$.

Kessler J \& Geerling C (2006): Profil Environnemental du Burkina Faso, rapport final. Université agronomique de Wageningen, Pays-Bas. http://ec.europa.eu/development/ icenter/

Koffi AA, Kouassi FA, Koua N'goran SBK \& Soro D (2014): Les Loranthaceae, parasites des arbres et arbustes : cas du département de Katiola, au nord de la Côte d'Ivoire. Int. J. Biol. Chem. Sci. 8(6): 2552-2559.

Koffi CK, Duoudi H \& GAuTIER D (2016): Landscape diversity and associated coping strategies during food shortage periods: evidence from the Sudano-Sahelian region of Burkina Faso. Reg Environ Change 16:1-12.

Lessmeister A, Schumann K, Lykke A-M, Heubach K, Thiombiano A \& HaHn K (2015): Substitution of the most important and declining wild food species in southeast Burkina Faso. Flora et Vegetatio Sudano-Sambesica, 18: 11-20.

MagurRan AE (2004): Measuring biological diversity. The Journal of the Torrey Botanical Society 131, (3): 277-278.

MEDD (Ministère de l'Environnement et du Développement Durable) (2011): Programme d'Investissement Forestier - Burkina Faso, volume 2, 130p.

Mori SA, Boom BM, De Carvalho AM, Dos Santos TS (1983): Southern Bahian moist forests. The Botanical Review 49 (2): 155-232.

Nacoulma BMi, Schumann K, Traoré S, BernhardtRömermann M, Hahn K, Wittig $\mathrm{R}$ \& Thiombiano A (2011): Impact of land-use on West African savanna vegetation: a comparison between protected and communal area in Burkina Faso. Biodiversity Conservation, 20: 3341-3362. DOI 10.1007/s10531-011-0114-0

Oú́droago A, Thiombiano A, Hahn-Hadjali K \& GuinKO S (2006): Diagnostic de l'état de dégradation des peuplements de quatre espèces ligneuses en zone soudanienne du Burkina Faso. Sécheresse 17 (4): 485 - 491.

R Development Core Team (2008): A language and environment for statistical computing. R Foundation for Statistical Computing, Vienna, Austria. ISBN 3-900051-07-0, URL http://www.R-project.org

Rabiou H, Iro Dan Guimbo I, Bationo BA, IssaharouMatchi I \& Mahamane A, (2016): État des populations naturelles de Vitellaria paradoxa Gaertn. C. F. Dans la zone soudanienne du Niger et du Burkina Faso (Afrique de l'Ouest). Rev. Ivoir. Sci. Technol., 28 : 428 - 441

SAndjong RCS, Ntoupka M, Vroumsia T \& Ibrahima A (2018): Caractérisation structurale de la végétation ligneuse du Parc National de Mozogo-Gokoro (Cameroun). Flora et Vegetatio Sudano-Sambesica, 21: 7-24. DOI: 10.21248/ fvss. 21.56

SAWAdogo L (2007): Etat de la biodiversité et la de production des ligneux du Chantier d'Aménagement Forestier 
du NAZINON après une vingtaine d'années de pratiques d'aménagement. Center for International Forestry Research, Bogor, Indonesia, $42 \mathrm{p}$.

SAWADOGO L (2009): Influence des facteurs anthropiques sur la dynamique de la végétation des forêts classées de Laba et de Tiogo en zone soudanéenne au Burkina Faso. Thèse doctorat d'Etat, Université de Ouagadougou, Burkina Faso, 142p.

Sawadogo L, Nygard R \& PAllo F (2002): Effect of livestock and prescribed fire on coppice growth after selective cutting of Sudanian savannah in Burkina Faso. Ann. For Sci., 59. 185-195.
TAnyi TF, Etongo D \& Rabdo A (2018): Assessing the sustainability of fuelwood production and its potential impact on REDD+ in Burkina Faso. International Journal of Environmental Studies, 75 (1): 186-200, DOI: $10.1080 / 00207233.2017 .1386435$

Yoni G \& Sedego S (2015): Rapport final mission d'évaluation technique, socio-économique, écologique et de gouvernance des Chantiers d'Aménagement Forestier $(\mathrm{CAF})$ de Bougnounou -Nebielianayou, de Cassou, du Nazinon et de Sapouy Bieha dans la région du Centre Ouest au Burkina Faso. Ministère de l'Environnement et des Ressources Halieutiques. Initiatives Conseils International. $101 \mathrm{p}$. 